\title{
NONDEGENERATE HIGHER DEGREE FORMS OVER DEDEKIND DOMAINS
}

\section{B. COLEMAN AND JOEL CUNNINGHAM}

ABSTRACT. It is determined which finitely generated projective modules over a Dedekind domain admit nondegenerate symmetric $r$-linear forms.

In [1] D. K. Harrison introduces a Grothendieck ring based on nondegenerate symmetric $r$-linear forms, $r \geq 2$, over a Noetherian commutative ring. The structure results are quite strong for $r \geq 3$.

In this note we determine which finitely generated projective modules over a Dedekind domain admit a nondegenerate $r$-linear form.

Definition. Let $R$ be a commutative ring and let $V$ denote a finitely generated $R$-module. A symmetric $r$-linear map $\theta: V^{r} \rightarrow R$ is said to be nonde. generate if the induced group homomorphism $\Gamma: V \otimes \cdots \otimes V \rightarrow \operatorname{Hom}_{R}(V, R)$ $=V^{*}$ given by $\Gamma\left(x_{1} \otimes \cdots \otimes x_{r-1}\right)(x)=\theta\left(x_{1}, \ldots, x_{r-1}, x\right)$ is surjective. In this case $(V, \theta)$ is called a nondegenerate space. Harrison's ring is the Grothendieck ring based on isomorphism classes of nondegenerate symmetric $r$-linear space under orthogonal direct sum and tensor product.

According to [1, Lemma 2.6], if $(V, \theta)$ is nondegenerate, then $V$ is projective; so let $R$ be a Dedekind domain and $V$ a finitely generated projective $R$-module. Thus $V \cong \mathfrak{U} \oplus R \oplus \cdots \oplus R$ for some ideal $\mathfrak{U}$ of $R$. If $V$ admits a nondegenerate bilinear form, then $\Gamma: V \rightarrow V^{*}$ induced by $\theta$ is an isomorphism, so we see that $\mathfrak{U} \cong \mathfrak{U}-1$, i.e. $\mathfrak{U}^{2}$ is principal. Conversely if $\mathfrak{U}^{2}=$ $\lambda R$ for some $\lambda$ in the field of fractions $K$ of $R$, then it is easy to see that $\theta(x, y)=x y / \lambda$ is a nondegenerate bilinear form on 2 . Thus $V$ admits a nondegenerate bilinear form if and only if $\mathfrak{Q}^{2}$ is principal. Similarly one argues that $\mathscr{U}$ admits a nondegenerate $r$-linear form iff $\mathfrak{U}^{r}$ is principal.

Now consider the case $r \geq 3$ with rank $V>1$. We claim that in this case $V$ will always admit a nondegenerate form $\theta$. It will suffice to consider $V=$ $\mathfrak{U} \oplus R$. Let $e_{1}, e_{2} \in K \oplus K$ be such that $V=\mathfrak{U} e_{1}+R e_{2}$. A symmetric $r$-linear form on $V$ corresponds precisely to elements $\mu_{0}, \mu_{1}, \ldots, \mu_{r}$, where $\mu_{k}$ $\epsilon \mathfrak{U ^ { - k }}, k=0, \ldots, r$ as follows: Given the $\mu_{k}$ we define $\theta$ on $K \oplus K$ by $\theta\left(e_{i_{1}}, \ldots, e_{i_{r}}\right)=\mu_{k}$ if exactly $k$ of the subscripts $i_{1}, \ldots, i_{r}$ are equal to 1. Then since $\mu_{k} \in \mathbb{U}^{-k}, \theta$ restricted to $V^{r}$ gives a mapping from $V^{r}$ into $R$.

Presented to the Society, January 17, 1974 under the title Forms on Dedekind domains; received by the editors July 29, 1974.

AMS (MOS) subject classifications (1970). Primary 16A50, 12 A99. form.

Key words and phrases. Dedekind domain, projective module, nondegenerate 
Lemma. Let $\theta$ be a symmetric $r$-linear form on $\mathfrak{A} \oplus R$ associated $w$ ith $\mu_{0}, \mu_{1}, \ldots, \mu_{r}$ as above. In order that $\theta$ be nondegenerate, it is necessary and sufficient that for any $a \in \mathfrak{U}^{-1}, x \in R$, the equations

$$
x_{r-1} \mu_{r}+x_{r-2} \mu_{r-1}+\cdots+x_{0} \mu_{1}=a
$$$$
x_{r-1} \mu_{r-1}+x_{r-2} u_{r-2}+\cdots+x_{0} \mu_{0}=x
$$

have simultaneous solutions with $x_{j} \in \mathfrak{Q} \mathfrak{X}^{j}, j=0, \ldots, r-1$.

Proof. It follows directly from the definition that $\theta$ is nondegenerate iff given $a \in \mathfrak{U}^{-1}, x \in R$ there are $a_{1}^{i} \cdots a_{r-1}^{i} \in \mathfrak{U}, z_{1}^{i}, \ldots, z_{r-1}^{i} \in R, i=1$, $\ldots, m$, such that

$$
\begin{aligned}
\left(\sum_{i=1}^{m} a_{1}^{i} \cdots a_{r-1}^{i}\right) \mu_{r}+\left(\sum_{j=1}^{r-1} \sum_{i=1}^{m} a_{1}^{i} \cdots \hat{a}_{j}^{i} \cdots a_{r-1}^{j}\right) z_{j}^{i} \mu_{r-1} \\
+\cdots+\left(\sum_{j_{1}<\cdots<j_{t}} \sum_{i=1}^{m} a_{1}^{i} \cdots a_{j_{1}}^{i} \cdots \hat{a}_{j_{2}}^{i} \cdots \hat{a}_{j_{t}}^{i} \cdots a_{r-1}^{i}\right) z_{j_{1}}^{i} \cdots z_{j_{t}}^{i} \mu_{r-t} \\
+\cdots+\left(\sum_{i=1}^{m} z_{1}^{i} \cdots z_{r-1}^{i}\right) \mu_{1}=a
\end{aligned}
$$

and

$$
\begin{aligned}
&\left(\sum_{i=1}^{m} a_{1}^{i} \cdots a_{r-1}^{i}\right) \mu_{r-1}+\left(\sum_{j_{1}<\cdots<j_{t}} \sum_{i=1}^{m} a_{1}^{i} \cdots \hat{a}_{j_{1}}^{i} \cdots \hat{a}_{j_{2}}^{i} \cdots \hat{a}_{j_{t}}^{i} \cdots a_{r-1}^{i}\right) \\
& z_{j_{1}}^{i} \cdots z_{j_{t}}^{i} \mu_{r-t-1}+\cdots+\left(\sum_{i=1}^{m} z_{1}^{i} \cdots z_{r-1}^{i}\right) \mu_{0}=x .
\end{aligned}
$$

(A caret over a symbol indicates its deletion.) Thus necessity is clear.

Now suppose we have a solution $x_{0}, \ldots, x_{r-1}$ to $(*)$, with $x_{j} \in \mathfrak{U}^{j}, j=$ $0, \ldots, r-1$. We show by induction that there are positive integers $M_{1}<M_{2}$ $<\ldots<M_{r+1}$, such that for each $t \leq r$ and each $s \leq t$,

$$
\sum_{j_{1}<\cdots<j_{s}} \sum_{i=1}^{M+1} a_{1}^{i} \cdots \hat{a}_{j_{1}}^{i} \cdots \hat{a}_{j_{2}}^{i} \cdots \hat{a}_{j_{s}}^{i} \cdots a_{r-1}^{i} z_{j_{1}}^{i} \cdots z_{j_{s}}^{i}=x_{r-s-1} .
$$

Choose $a_{1}^{i}, \ldots, a_{r-1}^{i} \in \mathscr{U}, i=1, \ldots, M_{1}$, such that $\sum_{i=1}^{M_{1}} a_{1}^{i} \cdots a_{r-1}^{i}=x_{r-1}$. Choose $z_{j}^{i}=0$ for $1 \leq j \leq r-1, i=1, \ldots, M_{1}$.

Now suppose $t \geq 1$ and $M_{1}<M_{2}<._{M}<M_{t}$ have been chosen along with $a_{1}^{i}, \ldots, a_{r-1}^{i} ; z_{1}^{i}, \ldots, z_{r-1}^{i}$ such that $\sum_{i=1}^{M} a_{1}^{i} \cdots a_{r-1}^{i}=x_{r-1}$ and

$$
\sum_{1 \leq k_{1}<\cdots<k_{j-1} \leq r-1} \sum_{i=1}^{M_{t}} a_{1}^{i} \cdots a_{k_{1}}^{i} \cdots \hat{a}_{k_{2}}^{i} \cdots \hat{a}_{k_{j-1}}^{i} z_{k_{1}}^{i} \cdots z_{k_{j-1}}^{i}=x_{r-j}
$$


Choose $M_{t+1}>M_{t}$ and for $i=M_{t}+1, \ldots, M_{t+1}$, choose $a_{t+1}^{i}, \ldots, a_{r-1}^{i}$ $\epsilon \mathfrak{A}$ such that

$$
\sum_{i=M_{t}+1}^{M+1} a_{j+1}^{i} \cdots a_{r-1}^{i}=x_{r-t-1} .
$$

Let $a_{1}^{i}=\cdots=a_{t}^{i}=0, z_{1}^{i}=\cdots=z_{t}^{i}=1, z_{t+1}^{i}=\cdots=z_{r-1}^{i}=0$ for $M_{t}<i \leq$ $M_{t+1}$. Then by induction,

$$
\sum_{i=1}^{M_{t+1}} a_{1}^{i} \cdots a_{r-1}^{i}=\sum_{i=1}^{M_{t}} a_{1}^{i} \cdots a_{t-1}^{i}=x_{r-1}
$$

and

$$
\begin{aligned}
\sum_{j_{1}<\cdots<j_{s} \leq r-1} & \sum_{i=1}^{M_{t+1}} a_{1}^{i} \cdots \hat{a}_{j_{1}}^{i} \cdots \hat{a}_{j_{2}}^{i} \cdots \hat{a}_{j_{s}}^{i} \cdots a_{r-1}^{i} z_{j_{1}}^{i} \cdots z_{j_{s}}^{i} \\
& =\sum_{j_{1}<\cdots<j} \sum_{i=1}^{M_{t}} a_{1}^{i} \cdots \hat{a}_{j_{1}}^{i} \cdots \hat{a}_{j_{2}}^{i} \cdots \hat{a}_{j_{s}}^{i} \cdots a_{r-1}^{i} z_{j_{1}}^{i} \cdots z_{j_{s}}^{i} \\
& =x_{r-s-1} \quad \text { for } s<t .
\end{aligned}
$$

Finally,

$$
\begin{aligned}
\sum_{j_{1}<\cdots<j_{t}} \sum_{i=1}^{M_{t}+1} a_{1}^{i} \cdots \hat{a}_{j_{1}}^{i} \cdots \hat{a}_{j_{2}}^{i} \cdots \hat{a}_{j_{t}}^{i} \cdots a_{r-1}^{i} z_{j_{1}}^{i} \cdots z_{j_{t}}^{i} \\
=\sum_{i=M_{t}+1}^{M_{t+1}} a_{t+1}^{i} \cdots a_{r-1}^{i}=x_{r-t-1} .
\end{aligned}
$$

Now the Lemma follows by induction, with $m=M_{r} \cdot \square$

We now suppose that $r \geq 3, a \in \mathfrak{U}^{-1}$ and $x \in R$, and show there are $\mu_{0}$, $\ldots, \mu_{r}$ with $\mu_{i} \in \mathfrak{I}^{-i}$ such that $(*)$ has a solution $x_{1}, \ldots, x_{r-1}$ with $x_{j} \epsilon$ $\mathfrak{U}^{j}$. To this end choose $\mu_{0}=1, \mu_{1}=\cdots=\mu_{r-2}=0$; and choose $\mu_{r-1} \in \mathfrak{U}^{1-r}$ and $\mu_{r} \in \mathfrak{U}^{-r}$ such that

$$
\mu_{r} \mathfrak{Z}^{r-1}+\mu_{r-1} \mathfrak{Q}^{r-2}=\mathfrak{U}^{-1}
$$

We can do this since, in a Dedekind domain, any nonzero element of an ideal can be extended to a two element generating set for that ideal. Let $x_{r-1} \in$ $\mathfrak{U}^{r-1}, x_{r-2} \in \mathfrak{U}^{r-2}$ be such that

$$
\mu_{r-1}^{x}+\mu_{r-1} x_{r-2}=\alpha .
$$

Then let $x_{0}=x-\mu_{r-1} x_{r-1}$. It is clear that $0, \ldots, 0, x_{r-2}, x_{r-1}$ is a solution to $(*)$.

We summarize these results as follows. 
Theorem. Let $R$ be a Dedekind domain and let $V$ be a finitely generated projective $R$-module of rank $n \geq 1$, and let $r \geq 2$. Suppose $V \cong \mathfrak{U} \oplus R \oplus \cdots$ $\oplus R$ where $\mathfrak{U}$ is a nonzero ideal in $R$. Then $V$ admits a nondegenerate sym. metric r-linear form iff

(1) $r=2$ and $\mathfrak{Q}^{2}$ is principal, or

(2) $r \geq 3, n=1$ and $\mathfrak{U}^{r}$ is principal, or

(3) $r \geq 3, n \geq 2$.

Note that $\mu_{0}, \ldots, \mu_{r}$ give a nondegenerate form on $\mathscr{U} \oplus R$ iff the homomorphism from $\mathfrak{U}^{r-1} \oplus \mathfrak{U}^{r-2} \oplus \ldots \oplus \mathfrak{U} \oplus R$ to $\mathfrak{U}^{-1} \oplus R$ given by the matrix

$$
\left(\begin{array}{cc}
\mu_{r} & \mu_{r-1} \cdots \mu_{1} \\
\mu_{r-1} & \mu_{r-2} \cdots \mu_{0}
\end{array}\right)
$$

is surjective.

The referee has pointed out that the Theorem applies in case $R=D[x]$, where $D$ is a Dedekind domain. See [2, Corollary (6.4)].

Finally, we answer a question raised by the referee, thanks to a conversation with Paul Eakin. Let $R$ be a Noetherian domain of Krull dimension $d$. If $\mathfrak{A}$ is a projective ideal and if $r \geq d+2$, then $\mathfrak{U} \oplus R$ has a nondegenerate symmetric form. To see this it suffices to produce $\mu_{r}, \mu_{r-1}, \ldots, \mu_{r-d}$, with $\mu_{j} \in \mathfrak{U}^{-j}$, such that $\mathfrak{U}^{r} \mu_{r}+\cdots+\mathfrak{U}^{r-d_{\mu}} \mu_{r-d}=R$. (For then these $\mu_{j}$, along with $\mu_{1}=0$ will give a solution for the first equation in $(*)$, with $x_{0}$ arbitrary. Then with $\mu_{0}=1$, we can choose $x_{0}$ so that the second equation holds.) Take $\mu_{r}=1$ and choose $\mu_{r-1}$ so that $\mathfrak{Q T}^{r-1} \mu_{r-1}$ is not contained in any of the finite number of minimal primes over $\mathfrak{U}^{r}$. This is possible since $\mathfrak{U}^{r-1}$ is invertible. Then $\mathfrak{U}^{r}+\mathfrak{U}^{r-1} \mu_{r-1}$ has height $\leq d-1$. If $\mathfrak{U}^{r}+\mathfrak{U}^{r-1} \mu_{r-1} \neq R$, choose $\mu_{r-2}$ so that $\mathfrak{Q}^{r-2} \mu_{r-2}$ is not contained in any of the minimal primes over $\mathfrak{U}^{r}+\mathfrak{U}^{r-1} \mu_{r-1}$. Then $\mathfrak{U}^{r}+\mathfrak{U}^{r-1} \mu_{r-1}+\mathfrak{U}^{r-2} \mu_{r-2}$ has height $\leq d-2$. Continuing in this manner, we get the desired $\mu_{r}, \ldots, \mu_{r-d}$.

\section{REFERENCES}

1. D. K. Harrison, A Grothendieck ring of higher degree forms, University of Oregon Math. Dept., 1973 (mimeographed preprint).

2. H. Bass, Algebraic K-theory, Benjamin, New York, 1968. MR $40 \# 2736$.

DEPARTMENT OF MATHEMATICS, UNIVERSITY OF KENTUCKY, LEXINGTON, KENTUCKY 40506

DEPARTMENT OF MATHEMATICS, UNIVERSIT Y OF TENNESSEE AT CHATTANOOGA, CHATTANOOGA, TENNESSEE 37401 